\title{
Sciatica in degenerative spondylolisthesis of the lumbar spine
}

\author{
S Maheshwaran, A M Davies, N Evans, P Broadley, V N Cassar-Pullicino
}

\section{Clinical history}

A 57 year old female presented with a one year history of low back pain and left sided sciatica. Her general health was unremarkable. Plain radiographs of the lumbar spine were abnormal (fig 1). These films revealed a spondylolisthesis at the L4/5 level. There was no evidence of an underlying spondylolysis and the degenerative L4/5 facet joints predisposed to the forward listhesis.

The patient was given a lumbar support and a course of physiotherapy. Although the symptoms improved transiently, they gradually became worse, obliging her to give up her work as a secretary, because of constant back pain. She was referred for an MRI scan of the lumbar spine for further assessment.

\section{Radiological findings}

Magnetic resonance imaging (MRI) of the lumbar spine was performed (Siemens 1 Tesla Impact) using sagittal and axial $\mathrm{T} 1$ and $\mathrm{T} 2$ weighted sequences. This revealed a soft tissue mass $1 \mathrm{~cm}$ in diameter on the medial aspect of the left $L 4 / 5$ facet joint causing expansion of the lateral recess of L5 (fig 2A). The mass exhibited intermediate signal intensity on the T1 weighted sequences (fig 2A), and high signal intensity on the $\mathrm{T} 2$ weighted sequences (fig 2B, C). The left $L 4 / 5$ facet joint was degenerate, with fluid evident within the joint as high signal intensity on the T2 weighted images (fig 2B).

Precontrast computed tomography (CT) demonstrated the degenerate facet joints with a vacuum phenomenon on the left and an expanded left lateral recess (fig 3A). Following the left L4/5 facet arthrogram, the CT showed contrast medium within the facet joint and the soft tissue mass (fig 3B).

\section{Diagnosis}

Lumbar intraspinal synovial cyst.

\section{Discussion}

The lumbar intraspinal synovial cyst is an uncommon cause of low back pain or sciatica. ${ }^{1}$ Since the advent of CT and MRI, the increasing number of reports indicates that they are not as rare as originally considered and reflects the improved diagnostic accuracy of diagnostic imaging. The case described above is typical in that the majority of these cysts (approximately $60-75 \%$ ) occur at the L4/5 level, with the remainder at the $\mathrm{L} 3 / 4$ and $\mathrm{L} 5 / \mathrm{S} 1$ levels. ${ }^{23}$ While the aetiology is unknown, it is
The MRI Centre, The Royal

Orthopaedic Hospital, Birmingham B31 2AP, United Kingdom S Maheshwaran

A M Davies

N Evans

Department of

Radiology,

The Robert Jones and Agnes Hunt

Orthopaedic Hospital, Oswestry, Shropshire, United Kingdom

P Broadley

$\star$ V N Cassar-Pullicino

Correspondence to:

Dr A M Davies.
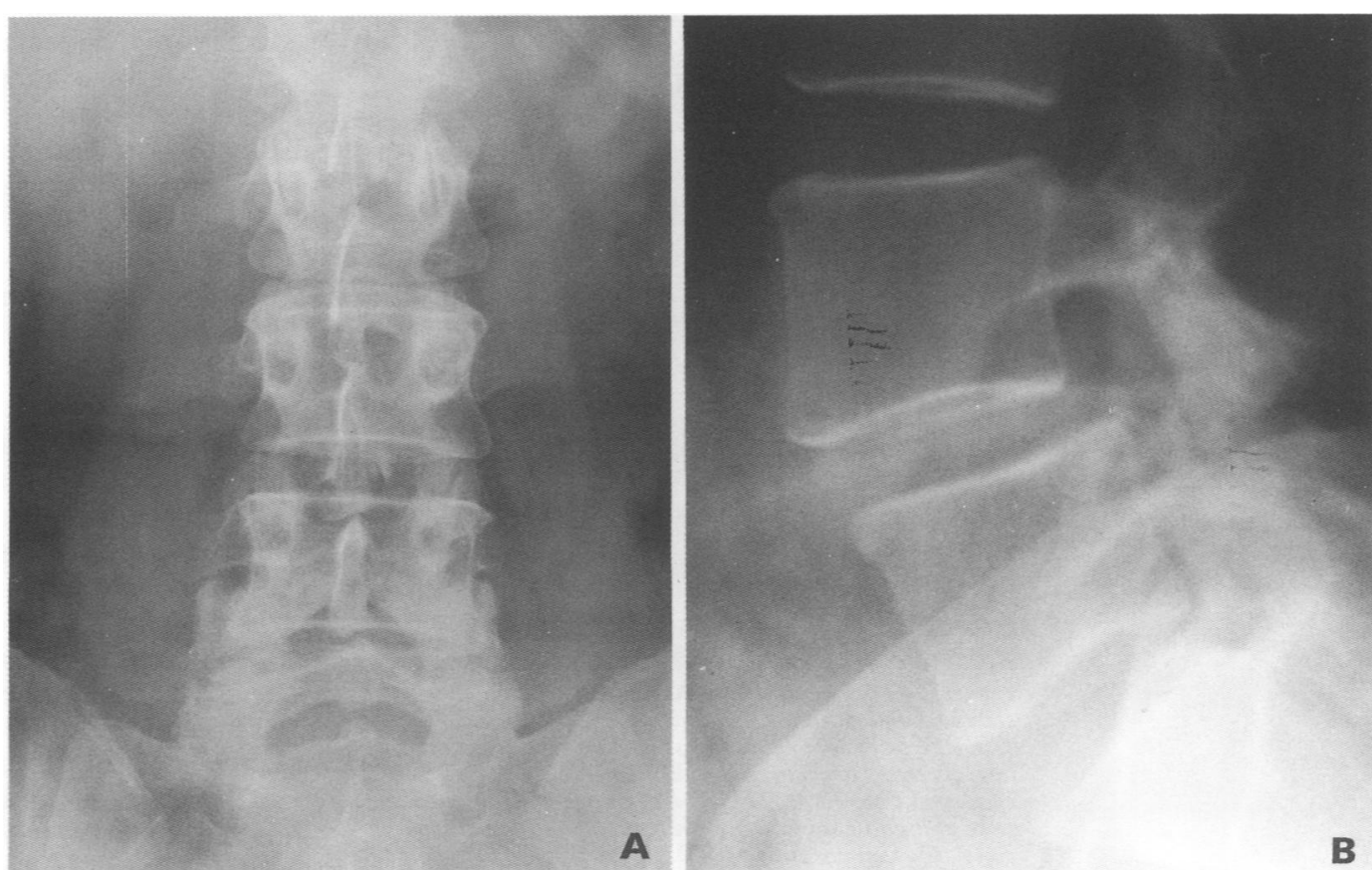

Figure 1 Present patient: Frontal and lateral radiographs of the lumbar spine showing degenerative spondylolisthesis at $L 4 / 5$. 

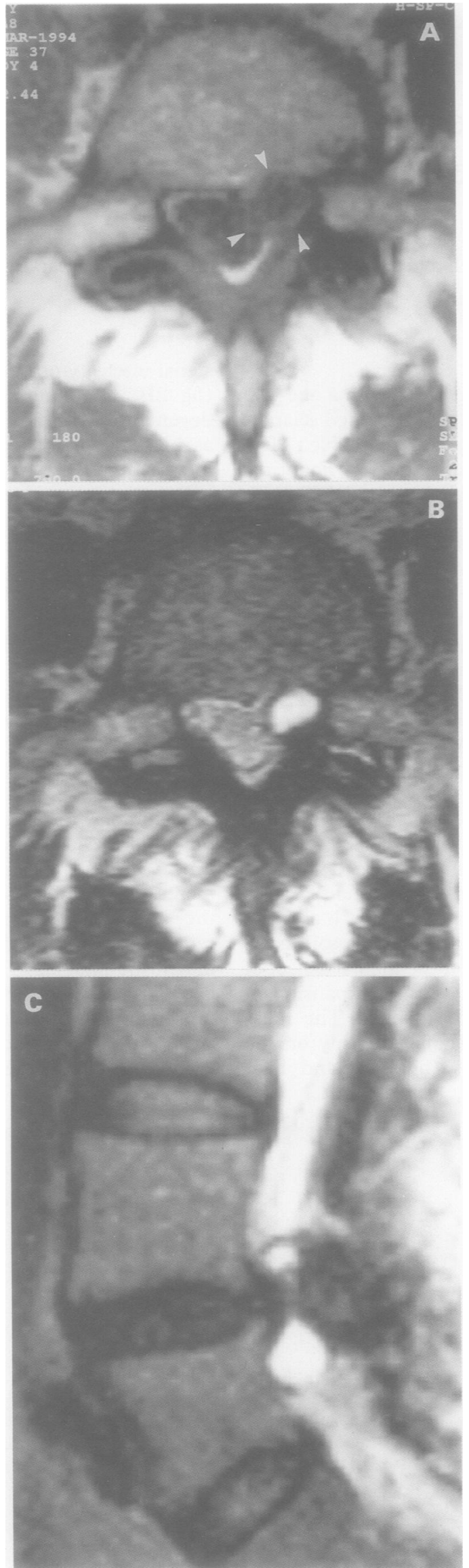

Figure 2 Present patient. A: Axial T1 weighted fast spin-echo image (repetition time (TR) $700 \mathrm{~ms}$, echo time (TE) $12 \mathrm{~ms}$ ) showing a soft tissue mass expanding the left lateral recess (arrowheads). B: Axial T2 weighted fast spin-echo (TR 3000, TE $158 \mathrm{~ms}$ ) image showing the mass to be of high signal intensity with a low signal intensity

rim. C: Left parasagittal T2 weighted fast spin-echo (TR $3000 \mathrm{~ms}$, TE $158 \mathrm{~ms}$ ) image showing the synovial cyst as a dumbell shaped lesion.
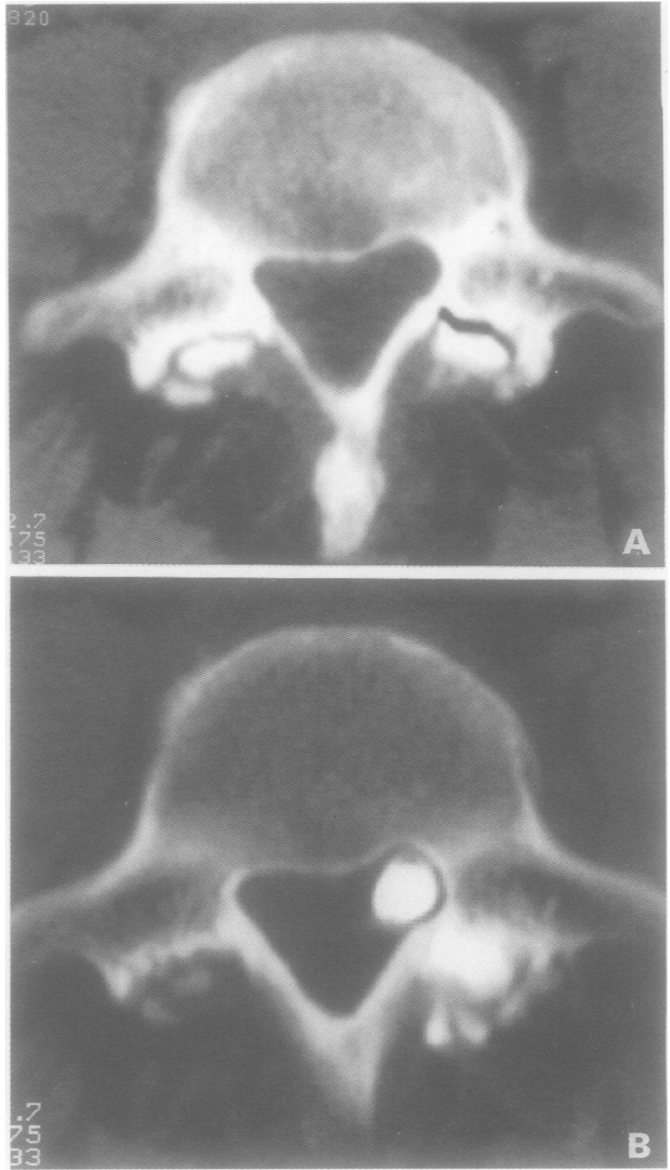

Figure 3 Present patient. A: Axial computed tomography (CT) showing expansion of the left lateral recess and a vacuum phenomenon in the facet joint. B: Axial CT after facet arthrography, showing the contrast medium within the facet joint and the synovial cyst.

generally thought to be degenerative, with osteoarthritis of the adjacent facet joint a universal finding, apart from the rare case secondary to trauma or inflammatory arthritis. ${ }^{45}$ The location of the cysts in the region of maximum spinal mobility and the association of degenerative change suggest that they may arise as a result of chronic irritation. ${ }^{3}$ The joint effusion bulges the joint capsule, and the synovial membrane herniates through the joint capsule-ligamentum flavum complex to form a synovial cyst. The term synovial cyst also refers to juxta-articular ganglion cysts which do not communicate with joints.

\section{CLINICAL FEATURES}

Most patients present with chronic back pain with or without sciatica (symptoms of degenerative disc and joint disease). The symptoms are of increasing intensity. The usual conservative measures of bed rest, physiotherapy, analgesics, and non-steroidal anti-inflammatory drugs (NSAIDs) fail to relieve the pain. ${ }^{6}$ There are some instances of spontaneous resolution of symptoms, probably as a result of collapse of the cyst. The occasional presentation with acute sciatica is indistinguishable from disc herniation. This has been attributed to the onset of haemorrhage into the cyst. 


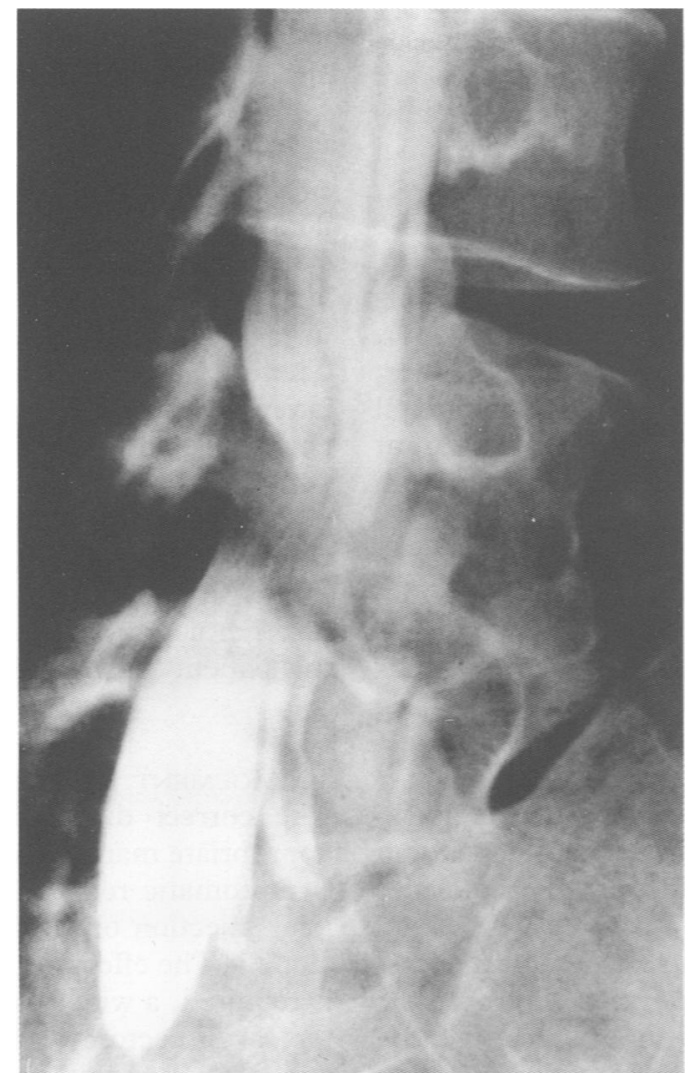

Figure 4 Example of a posterolateral compression of the lumbar theca seen in an oblique view of a myelogram.

\section{IMAGING FEATURES}

Plain radiographic changes are non-specific, but characteristically demonstrate a degenerative spondylolisthesis, as in this patient (fig 1). This is found in approximately $40 \%$ of patients. ${ }^{67}$ Although degenerative changes of the associated facet joint are seen in all documented CT cases, apart from the rare cases of post-traumatic origin or rheumatoid arthritis, the plain films may underestimate the facet joint changes. ${ }^{8}$

At myelography, a posterolateral extradural impression on the column of contrast medium is seen; figure 4 shows an example of this (not from the present patient). A similar appearance, however, may also be seen in hypertrophic facet joint degeneration. These changes differ from the anterior or anterolateral indentation caused by a disc herniation. ${ }^{8}$ The appearances are non-specific and would be consistent with any extradural spinal space occupying lesion at that site.

On CT, fortunately, the majority of these synovial cysts exhibit an attenuation value (greater than that of cerebrospinal fluid (CSF), but less than that of disc material) and are easily identified. The associated facet joint almost always shows degenerative changes. Increased attenuation may be seen if there has been haemorrhage into the cyst, or in the chronic stage when peripheral or internal calcification occurs. ${ }^{9}{ }^{10}$ The rim of the cyst wall maybe of high density attributable to either calcium deposition (fig 5C) or haemosiderin. Approximately $50 \%$ of the patients documented in the literature who had undergone CT examination had either a high density cyst wall
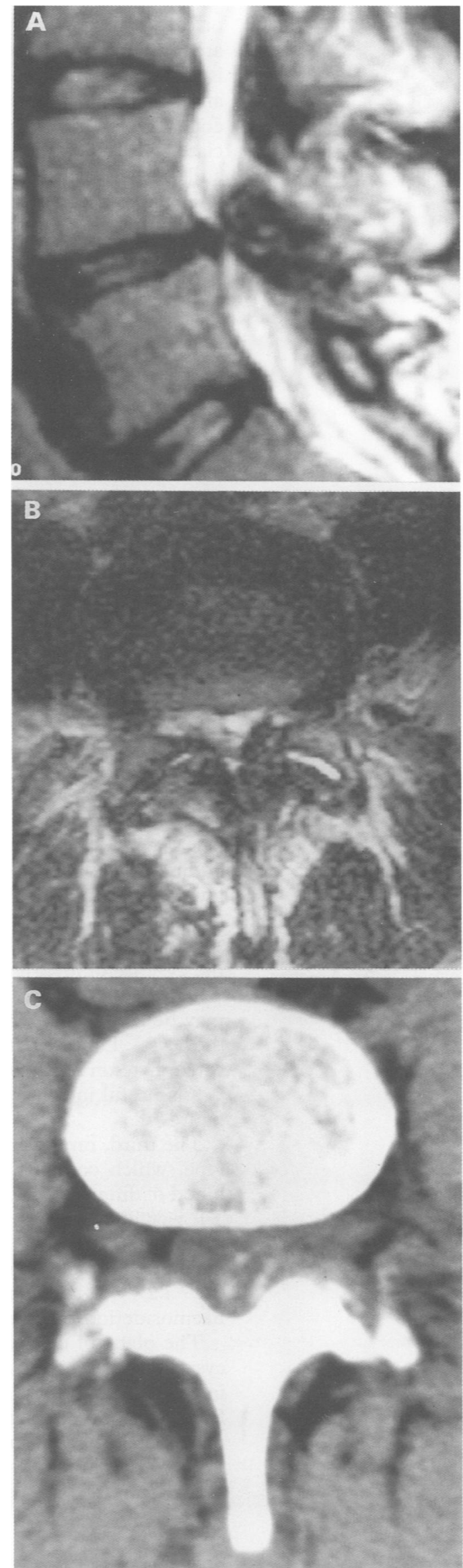

Figure 5 A: Left parasagittal T2 weighted fast spin-echo image (repetition time (TR) $4000 \mathrm{~ms}$, echo time (TE) $112 \mathrm{~ms}$ ) showing a lesion of heterogeneous signal intensity encroaching on the posterolateral theca at $L 4 / 5$, together with

spondylolisthesis. B: Axial T2 weighted fast spin-echo image (TR $4000 \mathrm{~ms}$, TE $112 \mathrm{~ms}$ ) showing bilateral facet joint osteoarthritis, with high signal intensity in the left facet joint and a low signal intensity mass between the facet and the theca. C: Axial CT showing calcification within the synovial cyst. 
or calcification within the cyst. ${ }^{8}$ Synovial cysts are difficult to diagnose on CT if they are isodense with the thecal sac. A negative attenuation can be found as a result of small collections of gas within the cyst, usually associated with a vacuum phenomenon in the adjacent facet joint. ${ }^{8}$

Facet arthrography can be useful in patients in whom the diagnosis remains in doubt, demonstrating the communication of the facet joint with the synovial cyst. ${ }^{10}$ Conventional radiography may not reveal the small amount of contrast medium within the cyst and CT, immediately after the arthrogram, is therefore required. ${ }^{8}$

The diagnosis on MRI is based on location, morphology, and signal (MR) characteristics. As demonstrated by $\mathrm{CT}$, these lesions are rounded, well circumscribed epidural masses related to the anteromedial aspect of the degenerate facet joint (fig 2B). ${ }^{311}$ The MR signal characteristics of the intraspinal synovial cyst are variable and depend on the fluid composition and the MR sequences used.

The MR signal characteristics of the cyst contents reflect its composition. Three basic patterns can be identified. In the first type, the non-haemorrhagic cyst, the signal intensity on the $T 1$ weighted spin-echo image is iso-intense with that of theca (fig $2 \mathrm{~A}$ ). The signal intensity on the $\mathrm{T} 2$ weighted images may be slightly hyperintense with respect to CSF (fig $2 \mathrm{~B}, \mathrm{C}$ ) because of increased protein content. In addition, the motion in CSF will provide a lower signal intensity than the static fluid in the synovial cyst. $^{3}$

The second pattern occurs when contents of the cyst are of low signal intensity on both the $T 1$ and $T 2$ weighted images (fig 5A,B). This may be caused by either air or calcification in the cyst. Differentiating this from a large osteophyte on MRI alone may prove difficult. ${ }^{3}$ CT is useful in this situation, as illustrated in figure $5 \mathrm{C}$.

The third, rarer, variety is the haemorrhagic type, which contains breakdown products of blood of differing ages. High signal intensity on $\mathrm{T} 1$ weighted images may be attributable to methaemoglobin as a result of subacute haemorrhage, and the low signal intensity on the T2 weighted images are caused by haemosiderin from chronic haemorrhage. ${ }^{3} 12$

The plane of cleavage between the synovial cyst and the CSF is seen as a low signal intensity rim and it clearly separates the CSF from the contents of the cyst and is seen well on the T2 weighted image in a type 1 cyst (fig $2 B, C)$. This rim may contain calcium which may be missed on MR but seen easily on CT. This is not the 'true rim' of the cyst and it appears to be the inflammatory interface between the cyst and the dura. Rim enhancement seen after intravenous injection of the paramagnetic contrast medium gadolinium-diethylene triamino pentacetic acid is characteristic. ${ }^{13}$ It was present in all five patients reported by Yuh et al, with over $50 \%$ also showing enhancement within one or both facet joints. ${ }^{11}$ All three patients given gado- linium in the series reported by Reginster $e t a l^{7}$ also showed rim enhancement.

\section{DIFFERENTIAL DIAGNOSIS}

The differential diagnosis of lumbar intraspinal synovial cyst includes conjoint nerve roots, a large herniated disc with free fragments, other intraspinal cysts, and cystic neurofibromata. These can be usually differentiated by the lateral location, associated degenerative changes, and the signal characteristics. Although bone erosion is a typical feature of neurofibromata, it may be present in any chronic process within the spinal canal with the propensity to enlarge. This includes chronic disc herniation, ${ }^{14} 15$ and synovial cysts ${ }^{10}$ as in this patient (figs $2 \mathrm{~A}, 3 \mathrm{~A}$ ).

\section{MANAGEMENT}

The correct diagnosis is important so that appropriate management can be determined. ${ }^{10}$ Symptomatic relief may be achieved by facet joint injection of local anaesthetic and steroid alone. ${ }^{16}$ The effect of this is variable. If surgery is required, a wider exposure than that for the typical discectomy is required to identify the adherent dura mater above and below the cyst in order to avoid surgical disruption of the dura. ${ }^{17}$ During laminectomy the cyst may collapse and the surgeon may not identify it. This may be a reason why synovial cysts were diagnosed infrequently before the availability of CT, as it is a non-specific diagnosis on myelography.

\section{Summary}

Intraspinal synovial cysts are an uncommon but well recognised cause of backache and sciatica, and should be considered in patients, in particular with degenerative spondylolisthesis, who are symptomatic. MRI is the initial investigation of choice. If there is any doubt as to the diagnosis, CT with or without facet joint arthrography is helpful.

1 Kiely M J. Neuroradiology case of the day: lumbar synovial cyst. Am $\mathcal{F}$ Roentgenol 1993; 160: 1336-7.

2 Liu S S, Williams K D, Drayer B P, Spetzler R F, Sonntag V K H. Synovial cysts of the lumbar spine: Diagnosis by MR imaging. Am f Roentgenol 1990; 154 163-6.

3 Jackson D E Jr, Atlas S W, Mani J R, Norman D. Intraspinal synovial cysts: MR imaging. Radiology 1989; 170: $527-30$

4 Silberbleit R, Gerbaraski S, Brunberg J, McGillicudy J, Blaivas M. Lumbar synovial cysts: correlation of myelographic, CT, MR and pathological findings. $A m \mathcal{F}$ myelographic, CT, MR and

5 Awwad E E, Sundaram M, Bucholz R D. Post traumatic spinal synovial cysts with spondylolysis: CT features. $\mathcal{f}$ spinal synovial cysts with spondylolysi

6 Reust P, Wendling D, Lagier R, et al. Degenerative spondylolisthesis, synovial cyst of the zygapophyseal joints and sciatic syndrome: report of 2 cases and review of the literature. Arthritis Rheum 1988; 31: 288-94

7 Reginster P, Collignon J, Dondelinger R F. Synovial cyst of the lumbar spine: CT and MRI correlations. Eur Radio 1994; 4: 332-6.

8 Knox A M, Fon G T. The appearance of lumbar intraspinal synovial cysts. Clin Radiol 1991; 44: 397-401.

9 Hemminghytt S, Daniels D L, Williams A L, Haughton $V$ M. Intraspinal synovial cysts: natural history and diagnosis by CT. Radiology 1982; 145: 375-6.

10 Lemish W, Apsimon T, Chakera T. Lumbar intraspinal synovial cysts: recognition and CT diagnosis. Spine 1989; 14: $1378-83$.

11 Yuh W T C, Drew J M, Weinstein J N, et al. Intraspinal synovial cysts: MR evaluation. Spine 1991; 16: 740-5. 
12 Summers R M, Quint D J. Case Report 712: Hemorrhagic synovial cyst arising from the right $\mathrm{L} 2 / \mathrm{L} 3$ facet joint

13 Wilms G, Hfkens L, Morlion J, et al. Bilateral lumbar intraspinal synovial cysts: Gd-enhanced MRI. Eur Radio 1993; 3: 471-3.

14 Vadala G, Dore R, Garbagna P. Unusual osseous changes in lumbar herniated discs: CT features. 7 Comput Assist Tomogr 1985; 9: 1045-49.
15 Flak B, Li D K B, Knickerbocker W J. Case Report 567 Chronic disc herniation causing bone erosion. Skeletal Radiol 1989; 18: 481-2.

16 Bjorkengren A G, Kurz L T, Resnick D, Sartoris D J, Garfin S R. Symptomatic intraspinal cysts; opacification and treatment by percutaneous injection. Am $\mathcal{F}$ Roentgenol 1987; 149: 105-7.

17 Onofrio B, Mih A. Synovial cysts of the spine. Neurosurgery 